CONTRIBUCIÓN DEL $3^{\text {ER }}$ CONGRESO LATINOAMERICANO DE MACROINVERTEBRADOS DE AGUA DULCE: BIODIVERSIDAD Y ECOLOGÍA FUNCIONAL EN EL NEOTRÓPICO

\title{
RIQUEZA Y DISTRIBUCIÓN DE ELMIDAE (INSECTA: COLEOPTERA: BYRRHOIDEA) EN EL DEPARTAMENTO DEL CHOCÓ, COLOMBIA
}

\author{
RICHNESS AND DISTRIBUTION OF ELMIDAE (INSECTA: COLEOPTERA: BYRRHOIDEA) \\ IN THE CHOCO DEPARTMENT FROM COLOMBIA
}

\author{
Marcela González-Córdoba, María del Carmen Zúñiga, Zuleyma Mosquera-Murillo y \\ Sandra Sánchez-Vásquez
}

\begin{abstract}
RESUMEN
Los élmidos son escarabajos acuáticos frecuentes y de amplia utilización como bioindicadores de calidad de agua por su sensibilidad a la contaminación orgánica. Para contribuir al conocimiento de la familia en el departamento del Chocó, región con estudios taxonómicos, ecológicos y de distribución sobre la familia incipientes, se revisaron 958 especímenes (adultos y larvas), depositados principalmente en la Colección Limnológica del Chocó (Universidad Tecnológica del Chocó) y el Museo de Entomología de la Universidad del Valle. Los especímenes corresponden a 14 municipios (entre 5 y $2063 \mathrm{msnm}$ ), 40 corrientes hídricas de diferente orden y dos ecosistemas lénticos que drenan principalmente a las cuencas de los ríos Atrato, San Juan y Baudó. Este trabajo registra 17 géneros (12 de la subfamilia Elminae y cinco de Larainae) y cuatro especies. Austrolimnius, Hexacylloepus, Hexanchorus, Huleechius, Microcylloepus, Neocylloepus, Neoelmis, Onychelmis, Stenhelmoides, Pharceonus, Pseudodisersus y Xenelmis, son nuevos registros para el Chocó y Cylloepus, Disersus, Heterelmis, Macrelmis y Phanocerus, ya conocidos, amplían su rango de distribución en la región. Austrolimnius pusio y A. formosus, son primeros registros para Colombia y Stenhelmoides rufulus y Pseudodisersus goudotti lo son para el Chocó. Estas citas representan el 70,8 \% de la riqueza genérica de Elmidae para Colombia y el mayor registro taxonómico se encontró en la cuenca media del río Atrato entre 25 y 150 msnm. La región Andina, el río Baudó y las cuencas altas de los ríos Atrato y San Juan, exhibieron los mayores vacíos de información. Macrelmis, Cylloepus, Microcylloepus, Phanocerus, Neoelmis y Heterelmis, presentaron la mayor distribución, mientras Disersus, Hexacylloepus, Onychelmis y Pharceonus, estuvieron restringidos a una sola localidad en zonas bajas. Con base en este trabajo el Chocó se ubica, conjuntamente con el Valle del Cauca, Antioquia y Meta, entre los departamentos de Colombia con la mayor riqueza genérica de la familia.
\end{abstract}

PALABRAS CLAVE: escarabajos acuáticos, Chocó biogeográfico, Pacífico, cuencas de los ríos Atrato, San Juan y Baudó

\begin{abstract}
The Elmidae are water beetles, common and widely used as bioindicators of water quality for their sensitivity to organic pollution. To contribute to the knowledge of the family in the department of Chocó, a region which the taxonomic, ecological and distributional studies for this family are incipient, specimens deposited mainly in the Colección Limnológica del Chocó (Universidad Tecnológica del Chocó) and the Museo de Entomología de la Universidad del Valle, were analyzed. 958 specimens (adults and larvae), collected in 14 municipalities between 5 and 2063 meters, 40 streams of different orders, and two lentic ecosystems that drain mainly into the Atrato and San Juan rivers were analyzed. 17 genera (12 of the subfamily Elminae and five of the Larainae) and four species are recorded in this study. Austrolimnius, Hexacylloepus, Hexanchorus, Huleechius, Microcylloepus, Neocylloepus, Neoelmis, Onychelmis, Stenhelmoides, Pharceonus, Pseudodisersus and Xenelmis, are new records to Chocó and Cylloepus, Disersus, Heterelmis, Macrelmis and Phanocerus, Cylloepus, Macrelmis and Heterelmis, already known, increase their range of distribution in the region. Two species are new records for Colombia (Austrolimnius pusio y A. formosus) and two for the Chocó (Stenhelmoides rufulus and Pseudodisersus goudotti). Those records represent $70.8 \%$ of the Elmidae generic richness known for Colombia. Most taxonomic records were found in the middle basin of the Atrato River between 25 and 150 meters. The Andean region and the upper basins of the Atrato and San Juan and the entire Baudó rivers exhibited the biggest lack information. Macrelmis, Cylloepus, Microcylloepus, Phanocerus, Neoelmis and Heterelmis, had the widest distribution, while Disersus, Hexacylloepus, Onychelmis and Pharceonus, were restricted to a single locality in low areas. Based on this work the Chocó, together with the Valle del Cauca, Antioquia and Meta, has the most generic richness of the family among the departments of Colombia.
\end{abstract}

KEY WORDS: water beetles, Biogeographical Choco, Atrato, Baudó and San Juan river basins 


\section{INTRODUCCIÓN}

Los élmidos son escarabajos acuáticos muy frecuentes y de amplia distribución en ambientes lóticos. Cumplen un importante papel en las redes tróficas de ecosistemas acuáticos y se utilizan como bioindicadores de calidad de agua por su sensibilidad al déficit de oxígeno y el incremento de la carga orgánica residual (Archangelsky et al., 2009; Zúñiga y Cardona, 2009). A nivel mundial se conocen alrededor de 1500 especies descritas en 150 géneros (Jäch et al., 2016). En Colombia se registran 22 géneros y 23 especies, distribuidas en 27 departamentos, de los cuales Antioquia, Valle del Cauca y Meta son los mejor representados en cuanto a riqueza genérica (González-Córdoba et al., 2015a, b; González-Córdoba, 2016).

El conocimiento sobre Elmidae en la región del Chocó es incipiente, y la mayoría de registros están a nivel de familia, principalmente porque sus registros se realizaron a partir de estudios de bioindicación de calidad de agua. Los órdenes Ephemeroptera y Trichoptera son los grupos de la entomofauna acuática que han recibido mayor atención en la región en aspectos taxonómicos, ecológicos y de distribución espacial y temporal (Camargo y Rozo, 2003; Asprilla et al., 2006; Bejarano et al., 2006; Mosquera et al., 2006; Casas-Córdoba et al., 2006; Córdoba et al., 2007; Mosquera-Murillo y Córdoba-Aragón, 2015a, b; Rozo y Salinas, 2016). Con base en estos trabajos, el registro genérico de Elmidae únicamente está representado por Cylloepus, Heterelmis y Macrelmis en la subfamilia Elminae y Disersus y Phanocerus en Larainae (Mosquera-Murillo y Córdoba-Aragón, 2015b), lo cual evidencia el vacío de información de esta familia en ambientes acuáticos de la región. Por las razones discutidas, este trabajo se realizó con el objetivo de contribuir al conocimiento de la representatividad taxonómica de Elmidae, con énfasis en su riqueza genérica y distribución geográfica y altitudinal en el departamento del Chocó y la ecorregión del Chocó biogeográfico.

\section{MATERIALES Y MÉTODOS}

\section{Área de estudio}

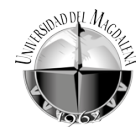

formada por los valles de los ríos Atrato y San Juan, y en su mayor parte está ocupada por selva ecuatorial, principalmente la del Darién que hace de límite natural con Panamá y marca la división entre América del Sur y América Central (Figura 1).

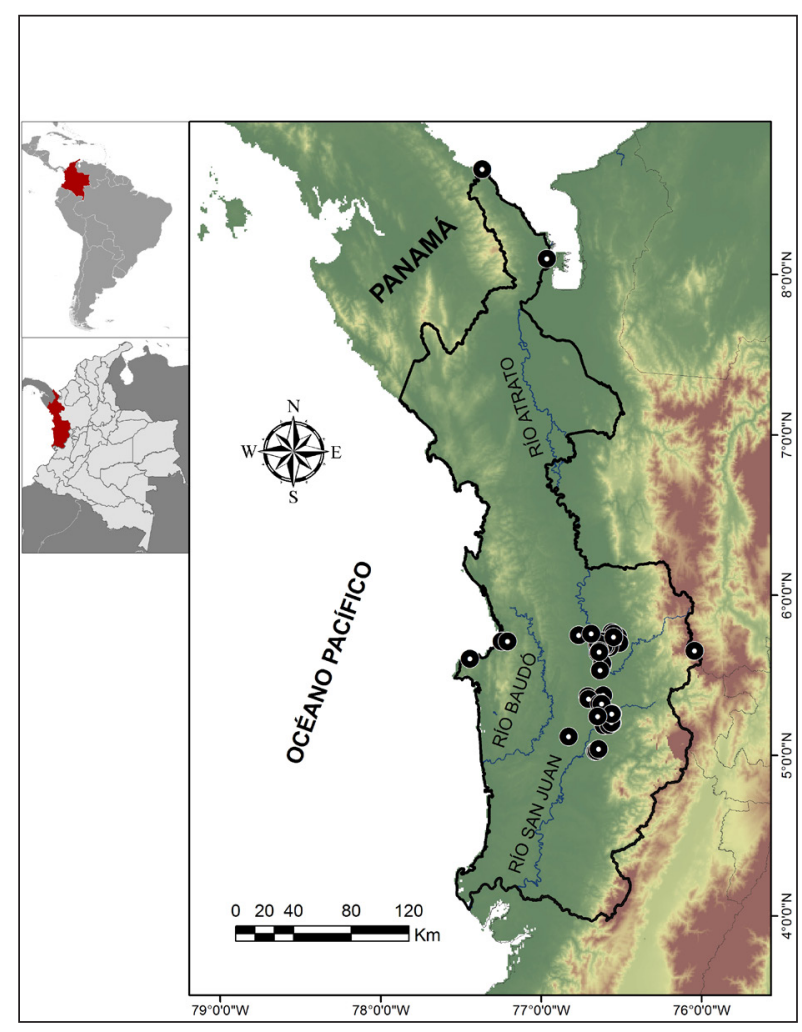

Figura 1. Representación geográfica de los muestreos de Elmidae en el departamento del Chocó.

El departamento está incluido en la ecorregión del Chocó biogeográfico, la cual se extiende desde la provincia panameña del Darién y la zona del golfo de Urabá en el norte, hasta la provincia de Manabí en sur oeste de Ecuador y desde el océano Pacífico hasta la divisoria de aguas en la vertiente occidental de la cordillera de los Andes (Rangel-Ch, 2004; Banco de Occidente, 2009). Esta región está identificada como un punto de alta diversidad ("hotspot") a nivel global, la cual posee una gran cantidad de endemismos (Mittermeier et al., 1998; Olson y Dinerstein, 1998; Myers et al., 2000; Rangel-Ch, 2004). El Chocó biogeográfico colombiano comprende la mitad del litoral nacional en el océano Pacífico y la zona de Urabá y representa alrededor del 70 \% de toda la ecorregión y el 10 \% del territorio nacional. Además, es una de las zonas con la mayor pluviosidad del planeta, 
lo cual genera una gran red hídrica, destacándose por su magnitud las cuencas de los ríos Atrato y San Juan (Banco de Occidente, 2009; IIAP, 2013).

El río Atrato es uno de los ríos más caudalosos del mundo en relación con su cuenca y uno de los más importantes del país. Nace en Carmen del Atrato en los Andes occidentales y cruza el departamento de sur a norte, alimentado por una amplia red fluvial conformada por alrededor de 150 ríos y 3000 microcuencas. Tiene una longitud de $750 \mathrm{~km}$, de los cuales $508 \mathrm{~km}$ son navegables por embarcaciones hasta de calado medio, un ancho variable entre 150 a $500 \mathrm{~m}$ y una profundidad de 31 a 38 m (Bedoya et al., 2009). Drena una cuenca de $38500 \mathrm{~m}^{2}$ y desemboca en el mar Caribe a través de un amplio delta de 18 bocas ubicado en el golfo de Urabá. Forma un amplio valle de tierras planas y anegadizas y varios caseríos y núcleos urbanos se asientan en la proximidad de sus riberas a lo largo de su curso. Su cuenca hidrográfica no es muy grande, pero al encontrarse en la zona de mayor precipitación pluvial de América, el aforo de su caudal se estima en $4900 \mathrm{~m}^{3} / \mathrm{s}$. Además, es considerada como una de las cuencas de mayor rendimiento a nivel mundial (Bedoya et al., 2009; IIAP, 2013).

Otra macrocuenca de importancia regional es la del río San Juan que nace en los Andes occidentales de Colombia, relativamente cerca de la cabecera del río Atrato, pero fluye del nororiente hacia el suroccidente en dirección opuesta al río Atrato, del cual está separado en la parte norte por una cadena de colinas con elevaciones de alrededor de $100 \mathrm{msnm}$. Desemboca en el océano Pacífico través de un delta de siete bocas que está situado a unos $60 \mathrm{~km}$ al noroeste del puerto de Buenaventura en el Valle del Cauca, e incluye numerosas islas rodeadas de manglares (Bedoya et al., 2009). A pesar de que la extensión de la cuenca se limita a $15000 \mathrm{~km}^{2}$, dada la abundancia de precipitaciones en la región, es la más importante de la vertiente del Pacífico colombiano y es el río más caudaloso que lleva sus aguas al océano Pacífico en Suramérica $\left(1300 \mathrm{~m}^{3} / \mathrm{s}\right)$. Esta corriente posee una longitud de $750 \mathrm{~km}$ y se ubica alrededor de $75 \mathrm{msnm}$, con temperaturas promedio entre 27 y 29 ${ }^{\circ} \mathrm{C}$. Posee una precipitación promedio multianual de $10000 \mathrm{~mm}$. Predominan las zonas de vida de bosque pluvial tropical (bp-T) y bosque muy húmedo tropical (bmh-T) (Holdridge, 1996; Bedoya et al., 2009; IIAP, 2013; Mosquera-Murillo y Córdoba-Aragón, 2015a).

\section{Revisión del material biológico}

Se revisaron ejemplares adultos y larvas recolectadas en cuerpos de agua del departamento del Chocó y depositados en la Colección Limnológica del ChocóCLCH (Universidad Tecnológica del Chocó), la Colección de Entomofauna Acuática del Museo de Entomología de la Universidad del Valle-MUSENUV y la Colección de Insectos Acuáticos de la Universidad Católica de Oriente-UCO. La identificación taxonómica se realizó en el Laboratorio de Investigaciones Entomológicas de la Universidad del Valle y se utilizaron las claves de Spangler y Santiago (1987); Spangler y Perkins (1989); Manzo (2005; 2006; 2007); Archangelsky et al. (2009); Miranda et al. (2012) y Sampaio et al. (2015). Las muestras se revisaron en un estereomicroscopio NIKON-SMZ 745 y para la determinación a nivel de especie se tuvieron en cuenta las estructuras genitales de ejemplares adultos machos, además de los caracteres taxonómicos discriminantes y definidos en las descripciones originales. El aparato genital masculino fue diseccionado y sometido a un proceso de aclaración con ácido láctico durante un período de dos semanas.

A partir de los datos de colecta y las coordenadas geográficas se elaboró el mapa de representación geográfica de los géneros. Para tal fin, se utilizó el software ARGIS $^{\circledR}$ versión 10.2 y adicionalmente se obtuvo un registro fotográfico de los principales géneros registrados, mediante un controlador de instrumentos Nikon DS-Ri1 U3 con una cámara de 12,7 megapixeles acoplada a un estereoscopio Nikon SMZ-1500 con 11,2 X de aumento.

\section{RESULTADOS}

Se revisaron 958 ejemplares (356 adultos y 602 larvas), recolectados en los ríos Cabí, Catugadó, Cértegui, Mungarrá, Pacurita, Pando, Pie de Pepe, Samurindó y Tutunendo, 31 corrientes hídricas de bajo orden 0 quebradas y dos ecosistemas lénticos (Ciénagas La Grande y Marriaga) (Figura 2). Estas corrientes drenan a los ríos Atrato, San Juan y Baudó y algunas pocas microcuencas pericontinentales fluyen directamente al mar Caribe o al Pacífico. Las estaciones de muestreo de los especímenes se ubicaron en los municipios de Acandí, Alto Baudó, Atrato, Bagadó, Cantón de San Pablo, Cértegui, Condoto, Istmina, Medio Baudó, Nuquí, Quibdó, Tadó, Unguía y Unión Panamericana, en un rango altitudinal entre 5 y $2063 \mathrm{msnm}$ y un período de 
muestreo entre 2003 y 2015. La Figura 1 representa la ubicación de las colectas que incluyen el 46,7 \% de los municipios del departamento y se observan vacíos de información en varias zonas, especialmente en el sector norte y sur. La mayoría de los datos corresponden a cuerpos de agua en municipios aledaños a Quibdó, la capital del departamento del Chocó, debido al acceso a las zonas que en muchos casos se dificulta por ser una región con predominio de selvas y falta de vías de comunicación.

La Tabla 1 compila el registro de géneros y especies de Elmidae identificadas en el Chocó y su distribución en términos de municipios, cuerpos de agua, rango altitudinal y macrocuencas de drenaje a las vertientes del Caribe o del Pacífico.

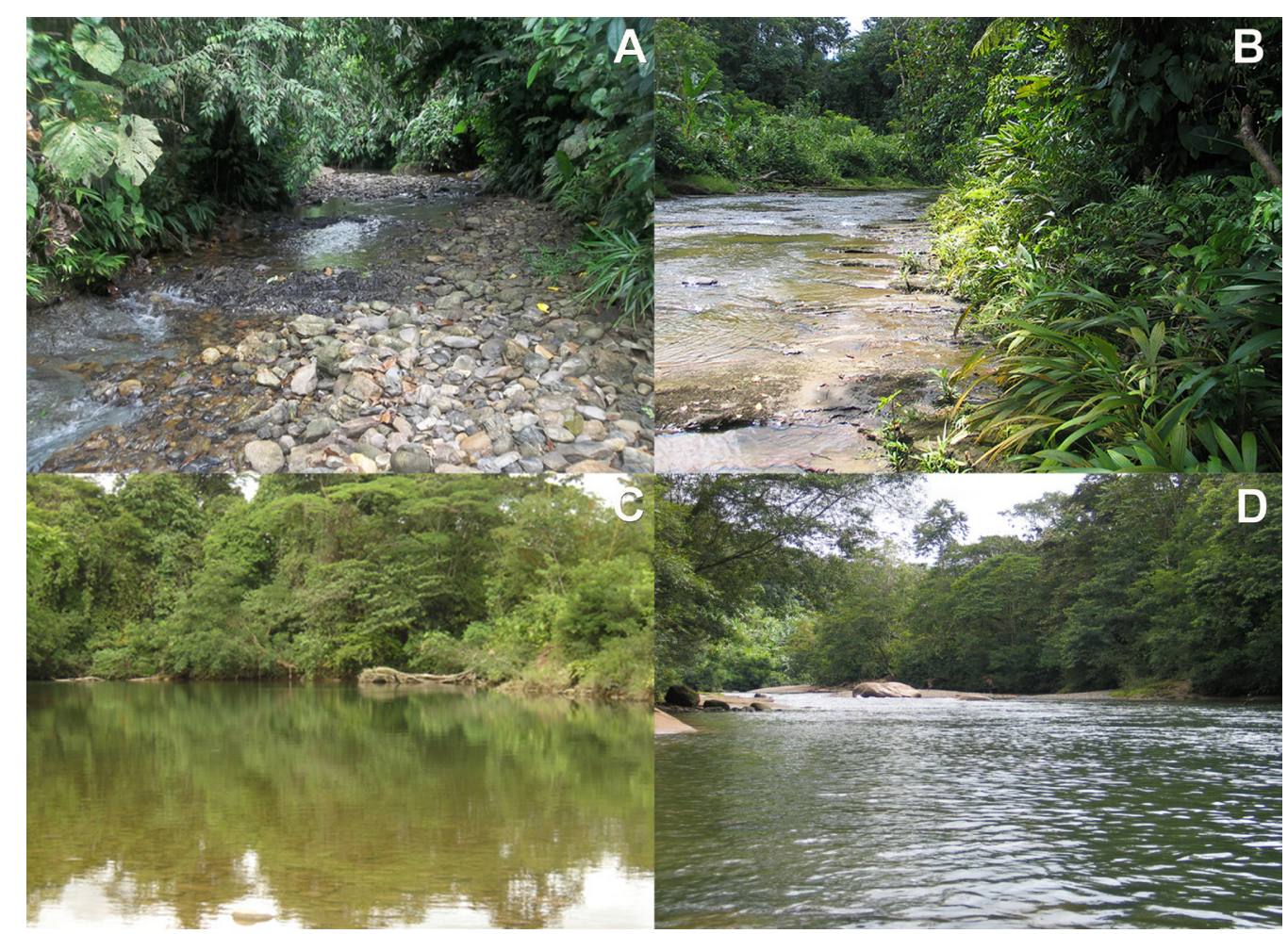

Figura 2. Corrientes hídricas en el departamento del Chocó. A) Quebrada San Bernabé. B) Río Catugadó. C) Río Mungarrá. D) Río Tutunendo. Fotos: Zuleyma Mosquera-Murillo

Tabla 1. Distribución de los géneros y especies de Elmidae en el departamento del Chocó.

\begin{tabular}{|c|c|c|c|c|}
\hline Género/Especie & $\begin{array}{l}\text { Rango de altura } \\
\text { (msnm) }\end{array}$ & Municipio & Cuerpo de agua & Macrocuenca \\
\hline $\begin{array}{l}\text { Austrolimnius* } \\
\text { Carter y Zeck, } 1929\end{array}$ & $39-96$ & Nuquí, Quibdó, Tadó & $\begin{array}{l}\text { Ríos: Cabí, Catugadó, Tutunendo } \\
\text { Quebradas: Santa Catalina, } \\
\text { Muertero }\end{array}$ & $\begin{array}{l}\text { Río Atrato } \\
\text { Río San Juan }\end{array}$ \\
\hline $\begin{array}{l}\text { A. formosus** } \\
\text { (Sharp, 1882) }\end{array}$ & $57-96$ & Quibdó & Ríos: Cabí, Tutunendo & Río Atrato \\
\hline $\begin{array}{l}\text { A. pusio** } \\
\text { Hinton, } 1941\end{array}$ & 57 & Quibdó & Río: Cabí & Río Atrato \\
\hline
\end{tabular}


Continuación Tabla 1.

\begin{tabular}{|c|c|c|c|c|}
\hline Género/Especie & $\begin{array}{l}\text { Rango de altura } \\
\text { (msnm) }\end{array}$ & Municipio & Cuerpo de agua & Macrocuenca \\
\hline $\begin{array}{l}\text { Cylloepus } \\
\text { Erichson, } 1847\end{array}$ & $25-138$ & $\begin{array}{l}\text { Atrato, Condoto, El Cantón } \\
\text { de San Pablo, Istmina, } \\
\text { Quibdó, Tadó, Unión } \\
\text { Panamericana. }\end{array}$ & $\begin{array}{l}\text { Ríos: Cabí, Guapandó, Catugadó } \\
\text { Mungarrá, Pando, Samurindo, } \\
\text { Tutunendo } \\
\text { Quebradas: Agua Clara, Chagarapa, } \\
\text { La Bendición, Los Monos, } \\
\text { Madrevieja, Oromenudo, Profundó, } \\
\text { Quitarrechera, Raspadurita, San } \\
\text { Francisco, Santa Catalina }\end{array}$ & $\begin{array}{l}\text { Río Atrato } \\
\text { Río San Juan }\end{array}$ \\
\hline $\begin{array}{l}\text { Disersus } \\
\text { Sharp, } 1882\end{array}$ & 83 & Tadó & Quebrada: Colorado & Río San Juan \\
\hline $\begin{array}{l}\text { Heterelmis } \\
\text { Sharp, } 1882\end{array}$ & $47-2063$ & $\begin{array}{l}\text { Bagadó, Istmina, Quibdó, } \\
\text { Tadó, Unión Panamericana. }\end{array}$ & $\begin{array}{l}\text { Ríos: Pacurita, Tutunendo } \\
\text { Quebradas: Alto de la Virgen, } \\
\text { Buena Vida, Guapandó, } \\
\text { Colorado, Oromenudo, Profundó, } \\
\text { Raspadurita, Santa Catalina. }\end{array}$ & $\begin{array}{l}\text { Río Atrato } \\
\text { Río San Juan }\end{array}$ \\
\hline $\begin{array}{l}\text { Hexacylloepus* } \\
\text { Hinton, } 1940\end{array}$ & 138 & Tadó & Quebrada: Profundó & Río San Juan \\
\hline $\begin{array}{l}\text { Hexanchorus* } \\
\text { Sharp, } 1882\end{array}$ & $5-103$ & $\begin{array}{l}\text { Quibdó, Unguía, Unión } \\
\text { Panamericana. }\end{array}$ & $\begin{array}{l}\text { Ríos: Catugadó, Guapando, } \\
\text { Tutunendo } \\
\text { Cuerpo de agua léntico: Ciénaga } \\
\text { Marriaga }\end{array}$ & $\begin{array}{l}\text { Río Atrato } \\
\text { Río San Juan }\end{array}$ \\
\hline $\begin{array}{l}\text { Huleechius* } \\
\text { Brown, 1981b }\end{array}$ & $60-127$ & Atrato, Cértegui, Quibdó & $\begin{array}{l}\text { Ríos: Samurindó, Tutunendo } \\
\text { Quebradas: Agua Clara, Platinero, } \\
\text { Cértegui. }\end{array}$ & Río Atrato \\
\hline $\begin{array}{l}\text { Macrelmis } \\
\text { Motschulsky, } 1859\end{array}$ & $37-150$ & $\begin{array}{l}\text { Atrato, Cantón de San } \\
\text { Pablo, Cértegui, Condoto, } \\
\text { El Cantón del San Pablo, } \\
\text { Istmina, Quibdó, Tadó, } \\
\text { Unión Panamericana. }\end{array}$ & $\begin{array}{l}\text { Ríos: Catugadó, Cabí, Cértegui, } \\
\text { Mungarrá, Pacurita, Pando, } \\
\text { Samurindó Tutunendo } \\
\text { Quebradas: Agua Clara, Buena } \\
\text { Vida, Chagarapa, Colorado, Doña } \\
\text { Josefa, El Caraño, Guapandó, } \\
\text { La Francisca, La Bendición, } \\
\text { Platinero, Profundó, Quitarrechera, } \\
\text { Raspadurita, Salerito, San José, } \\
\text { Santanita. }\end{array}$ & $\begin{array}{l}\text { Río Atrato } \\
\text { Río San Juan }\end{array}$ \\
\hline
\end{tabular}


Continuación Tabla 1.

\begin{tabular}{|c|c|c|c|c|}
\hline Género/Especie & $\begin{array}{l}\text { Rango de altura } \\
\text { (msnm) }\end{array}$ & Municipio & Cuerpo de agua & Macrocuenca \\
\hline $\begin{array}{l}\text { Microcylloepus* } \\
\text { Hinton, } 1935\end{array}$ & $37-150$ & $\begin{array}{l}\text { Atrato, Cantón de San } \\
\text { Pablo, Condoto, Istmina, } \\
\text { Quibdó, Tadó, Unión } \\
\text { Panamericana. }\end{array}$ & $\begin{array}{l}\text { Ríos: Catugadó,Tutunendo } \\
\text { Quebradas: Buena Vida, } \\
\text { Chagarapa, Colorado, Doña Josefa, } \\
\text { La Francisca, La Yesca, Los Monos, } \\
\text { Profundó, Raspadurita, Salerito, } \\
\text { Santa Catalina, San Bernabé. }\end{array}$ & $\begin{array}{l}\text { Río Atrato } \\
\text { Río San Juan }\end{array}$ \\
\hline $\begin{array}{l}\text { Neocylloepus* } \\
\text { Brown, } 1970\end{array}$ & $90-138$ & Tadó & $\begin{array}{l}\text { Río: Mungarrá } \\
\text { Quebradas: Buena Vida, Profundó }\end{array}$ & Río San Juan \\
\hline $\begin{array}{l}\text { Neoelmis* } \\
\text { Musgrave, } 1935\end{array}$ & $15-138$ & $\begin{array}{l}\text { Acandí, Alto Baudó, } \\
\text { Atrato, Condoto, Nuquí, } \\
\text { Quibdó, Quibdó, Tadó, } \\
\text { Unión Panamericana. }\end{array}$ & $\begin{array}{l}\text { Ríos: Buena Vida, Cabí, Catugadó, } \\
\text { Guapando, Samurindó } \\
\text { Quebradas: El Cielo, La Yesca, } \\
\text { Madrevieja, Muertero, Profundó, } \\
\text { Quitarrechera, Santa Catalina }\end{array}$ & $\begin{array}{l}\text { Caribe }+ \\
\text { Pacífica }+ \\
\text { Río Atrato } \\
\text { Río San Juan }\end{array}$ \\
\hline $\begin{array}{l}\text { Onychelmis* } \\
\text { Hinton, } 1941\end{array}$ & 96 & Quibdó & Río: Catugadó & Río Atrato \\
\hline $\begin{array}{l}\text { Phanocerus } \\
\text { Sharp, } 1882\end{array}$ & $15-138$ & $\begin{array}{l}\text { Acandí, El Cantón de San } \\
\text { Pablo, Nuquí, Quibdó, } \\
\text { Tadó. }\end{array}$ & $\begin{array}{l}\text { Río: Tutunendo } \\
\text { Quebradas: Buena Vida, } \\
\text { Chagarapa, Colorado, El Caraño, } \\
\text { El Cielo, Profundó, Quitarrechera, } \\
\text { Santa Catalina, Termales. } \\
\text { Cuerpo de agua léntico: Ciénaga La } \\
\text { Grande }\end{array}$ & $\begin{array}{l}\text { Caribe }+ \\
\text { Pacífica }+ \\
\text { Río Atrato } \\
\text { Río San Juan }\end{array}$ \\
\hline $\begin{array}{l}\text { Pharceonus* } \\
\text { Spangler y Santiago- } \\
\text { Fragoso, } 1992\end{array}$ & 15 & Acandí & Quebrada: El Cielo & Caribe + \\
\hline $\begin{array}{l}\text { Pseudodisersus* } \\
\text { Brown, 1981a }\end{array}$ & 96 & Atrato, Quibdó & $\begin{array}{l}\text { Río: Tutunendo } \\
\text { Quebrada: Mabarbara }\end{array}$ & Río Atrato \\
\hline $\begin{array}{l}\text { P. goudotti* } \\
\text { Spangler y Santiago, } \\
1987\end{array}$ & 96 & Quibdó & Río: Tutunendo & Río Atrato \\
\hline $\begin{array}{l}\text { Stenhelmoides* } \\
\text { Grouvelle, } 1908\end{array}$ & $32-96$ & Medio Baudó, Quibdó & Ríos: Pie de Pepe, Tutunendo & $\begin{array}{l}\text { Río Atrato } \\
\text { Río Baudó }\end{array}$ \\
\hline
\end{tabular}


Continuación Tabla 1.

\begin{tabular}{|c|c|c|c|c|}
\hline Género/Especie & $\begin{array}{l}\text { Rango de altura } \\
\text { (msnm) }\end{array}$ & Municipio & Cuerpo de agua & Macrocuenca \\
\hline $\begin{array}{l}\text { S. rufulus* } \\
\text { Hinton, } 1934\end{array}$ & 96 & Quibdó & Río: Tutunendo & Río Atrato \\
\hline $\begin{array}{l}\text { Xenelmis* } \\
\text { Hinton, } 1936\end{array}$ & $47-96$ & Quibdó & Ríos: Cabí, Catugadó, Pacurita & Río Atrato \\
\hline
\end{tabular}

\section{DISCUSIÓN}

El inventario taxonómico de Elmidae en este estudio incluye el registro de 17 géneros; 12 de la subfamilia Elminae (Austrolimnius, Cylloepus, Heterelmis, Hexacylloepus, Huleechius, Macrelmis, Microcylloepus, Neocylloepus, Neoelmis, Onychelmis, Stenhelmoides y Xenelmis) y cinco de Larainae (Disersus, Hexanchorus, Phanocerus, Pharceonus y Pseudodisersus) (Figura 3).

Cylloepus, Disersus, Heterelmis, Macrelmis y Phanocerus, cuya presencia en cuatro microcuencas que drenan al río San Juan fue citada recientemente por MosqueraMurillo y Córdoba-Aragón (2015b), en este trabajo amplían sus rangos de distribución en varias localidades que incluyen esta misma cuenca y afluentes adicionales que drenan a la cuenca del río Atrato (Tabla 1). Los 12 géneros restantes se constituyen en nuevos registros de distribución para el departamento.

La riqueza que documenta este estudio representan el 70,8 \% de los géneros de Elmidae a nivel nacional (González-Córdoba, 2016). La mayor representación taxonómica se encontró entre 5 y 150 msnm en la cuenca media del río Atrato (región Pacífica), mientras que la región Andina y, en particular, las cuencas altas de los ríos Atrato, San Juan y toda la cuenca del Baudó exhibieron los mayores vacíos de información (Figura 1).

En su orden, Neoelmis, Macrelmis, Microcylloepus, Cylloepus y Heterelmis, presentaron los mayores rangos de distribución en el departamento, lo cual coincide con la amplia distribución que muestran en diferentes regiones del país, no sólo a nivel geográfico, sino también altitudinal (Caupaz-Flórez et al., 2006;
Arias-Díaz et al., 2007; González-Córdoba et al., 2015a; González-Córdoba, 2016). En contraposición, Disersus, Hexacylloepus, Onychelmis y Pharceonus, estuvieron restringidos a una sola localidad en las zonas bajas que, en general presentan baja frecuencia de registro, pero amplio ámbito de distribución en Colombia (Tabla 1).

En cuanto a la distribución altitudinal, a pesar de que el rango general incluyó datos entre el nivel del mar y un poco más de $2000 \mathrm{msnm}$, la mayoría de los registros representan las zonas bajas, hasta los 150 msnm. Así mismo, los rangos de distribución altitudinal de los géneros fueron estrechos y los más amplios corresponden a Heterelmis, Macrelmis, Neoelmis y Microcylloepus, patrón que se repite en otras regiones para estos géneros (Tabla 1) (González-Córdoba et al., 2015a; González-Córdoba, 2016).

Los microhábitats en donde más se encontraron individuos en los eventos de muestreo fueron las piedras y la hojarasca, los cuales aparentemente ofrecieron mayor estabilidad, oferta alimenticia y refugio a los élmidos, a diferencia del sedimento que evidenció no ser un hábitat afín con este grupo de insectos acuáticos en estas localidades.

Con base en los resultados de este trabajo, el departamento del Chocó se ubica, conjuntamente con el Valle del Cauca, Antioquia y Meta, entre los departamentos de Colombia con la mayor riqueza genérica de la familia (González-Córdoba et al., 2015a, b; González-Córdoba, 2016). Aunque la riqueza de élmidos en los tres departamentos es igual (17 géneros), la composición en cada caso es diferente. Entre los géneros que de manera frecuente se comparten entre los diferentes departamentos de Colombia y regiones naturales, se encuentran Macrelmis, 
Heterelmis, Neoelmis, Microcylloepus, Cylloepus, Phanocerus, Pharceonus y Austrolimnius, mientras que Hexacylloepus, Neocylloepus, Stenhelmoides y
Xenelmis, que están pobremente representados o son de distribución restringida en el país, fueron encontrados en el departamento del Chocó (González- Córdoba, 2016).

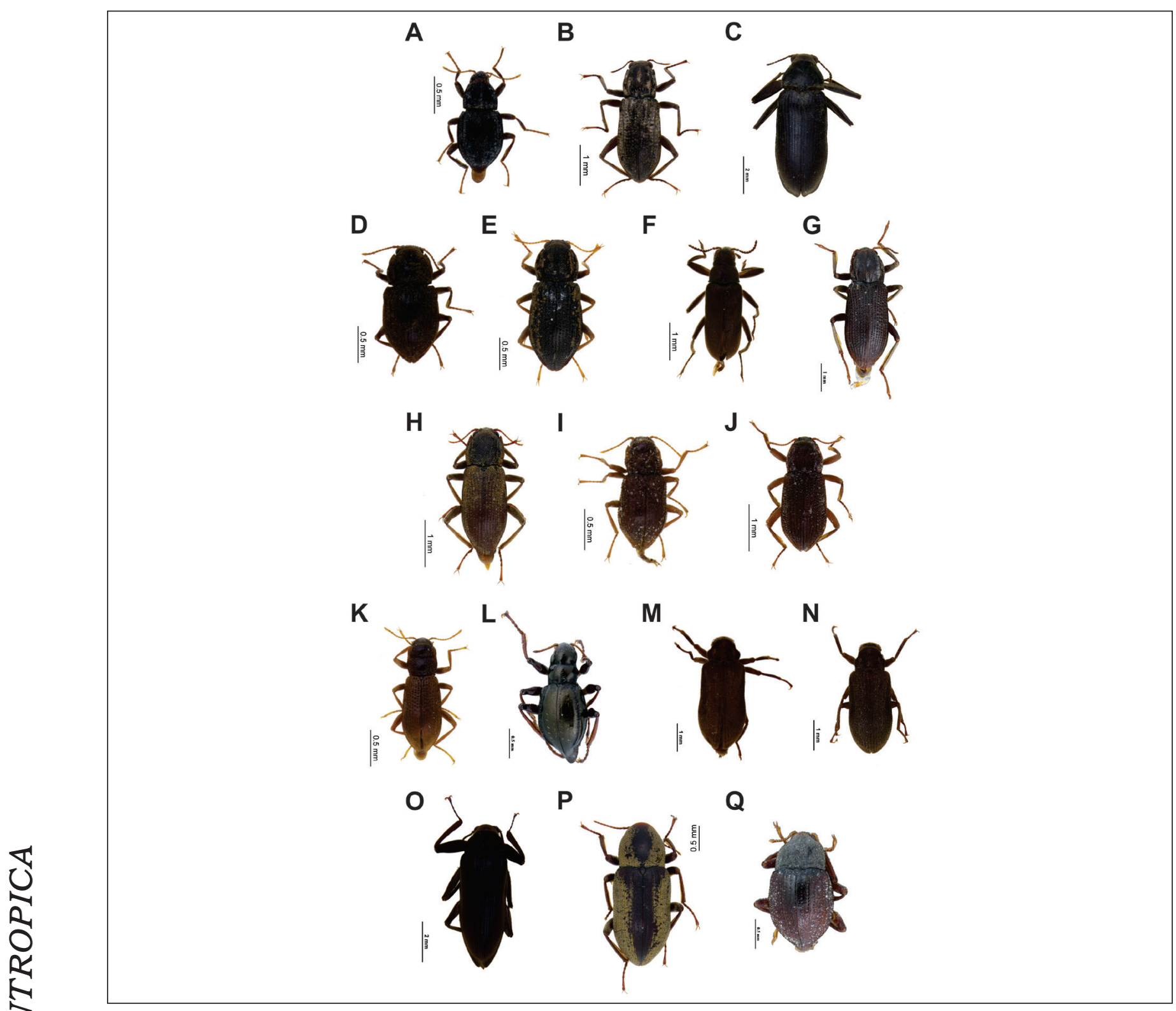

Figura 3. Elmidae del departamento del Chocó. Hábito dorsal de: A. Austrolimnius, B. Cylloepus, C. Disersus, D. Heterelmis, E. Hexacylloepus, F. Hexanchorus, G. Huleechius, H. Macrelmis, I. Microcylloepus, J. Neocylloepus, K. Neoelmis, L. Onychelmis, M. Phanocerus, N. Pharceonus, O. Pseudodisersus, P. Stenhelmoides y Q. Xenelmis. Fotos: Felipe Ortega. Laboratorio de Imágenes (Programa de Posgrado en Biología), Universidad del Valle.

Adicionalmente, se identificaron cuatro especies: Austrolimnius pusio Hinton, 1941, A. formosus Sharp, 1882 y Stenhelmoides rufulus Hinton, 1934 en Elminae y Pseudodisersus goudotti Spangler y Santiago, 1987 en Larainae. A. pusio y A. formosus, además de ser los primeros registros específicos para el Chocó, también son nuevos registros para Colombia, lo cual incrementa a 25 las especies registradas hasta la fecha para el país (González-Córdoba et al., 2015a, 2016). Las otras dos especies, ya conocidas en Colombia, amplían su rango de distribución para el Chocó. 
De las especies americanas de Austrolimnius, A. formosus exhibe el mayor rango de distribución; se encuentra desde el norte de Argentina hasta el sur de México y se diferencia de otras especies por presentar dos hileras de gránulos en la epipleura, tercer intervalo elitral plano, un estrechamiento de la impresión media hacia la mitad del pronoto en una razón de 1:2 con respecto a los extremos y el aparato genital masculino característico (Manzo, 2007). A. pusio se conoce en Brasil y Ecuador y se caracteriza por su tamaño reducido, la ausencia de la depresión media longitudinal que presentan muchas de las especies de Austrolimnius, las hileras de gránulos en la epipleura característicos del género se extienden hasta el ápice, además, no exhibe tubérculos en los trocánteres posteriores (Manzo, 2007).

Pseudodisersus goudotti se encuentra registrado en Colombia, Costa Rica, Ecuador y Panamá y es la única especie descrita del género, el cual se caracteriza por su gran tamaño $(10 \mathrm{~mm}$ ), por la ausencia de tomento (como la subfamilia Larainae), integumento café oscuro a negro muy pubescente, morfología del pronoto característica con líneas arqueadas a los lados, tubérculos con forma de papila en los ápices posterolaterales y frente al escutelo. El material tipo de la especie es de Colombia, registrado únicamente en Bogotá, así que este trabajo amplía su rango de distribución hasta la región del Pacífico colombiano (Spangler y Santiago, 1987).

Stenhelmoides rufulus conocido en Belice, Colombia, Costa Rica, Ecuador, Guatemala, Honduras, Panamá, Perú y Venezuela, es la especie más ampliamente distribuidas del género Stenhelmoides. Se diferencia de las otras especies por presentar tomento en el metafémur, cepillos limpiadores extendidos en tres cuartos de la protibia, una franja ovoide sin tomento en el centro del pronoto, así como dos hileras completas de puntos en los élitros. A pesar de que esta especie ya estaba registrada en los departamentos de Antioquia, Cesar, Putumayo y Valle de Cauca, en este trabajo se amplía su rango de distribución al departamento de Chocó (Spangler y Perkins, 1989).

La gran red hídrica de la ecorregión del Chocó biogeográfico y su riqueza biológica generan condiciones muy favorables para el desarrollo de la comunidad de coleópteros acuáticos como los Elmidae. A pesar de los vacíos de información que se evidenciaron en el trabajo, el registro de los géneros y especies que se documentan, ubican la región entre las de mayor riqueza genérica de Colombia. En tal sentido, es importante continuar con este tipo de estudios, enfocando los esfuerzos hacia aquellas zonas con información deficiente o inexistente, lo cual permitirá avanzar en el conocimiento de esta familia no sólo a nivel regional sino también del Neotrópico.

\section{AGRADECIMIENTOS}

A la Universidad Tecnológica del Chocó y en especial a la Colección Limnológica del Chocó por el soporte logístico y económico y por facilitar ejemplares. Al Museo de Entomología y a los Laboratorios de Investigaciones Entomológicas y de Imágenes de la Universidad del Valle (Programa de Posgrado en Biología), por la infraestructura y apoyo en la identificación taxonómica del material, en especial a Felipe Ortega por el registro fotográfico. A Luisa Fernanda Álvarez por facilitar algunos ejemplares de la Colección de Insectos Acuáticos de la Universidad Católica de Oriente. A todas aquellas personas que dieron apoyo en diferentes etapas del estudio, actividades de laboratorio y trabajo de campo para la recolecta de especímenes.

\section{BIBLIOGRAFÍA}

Archangelsky, M., Manzo, V., Michat, M.C. y Torres, P.M.L. 2009. Coleoptera. En: Domínguez, E. y Fernández, H., Editores. Macroinvertebrados bentónicos sudamericanos. Sistemática y Biología. Fundación Miguel Lillo, San Miguel de Tucumán, Argentina.

Arias-Díaz, D.M., Reinoso-Flórez, G., Guevara-Cardona, G. y Villa-Navarro, F.A. 2007. Distribución espacial y temporal de los coleópteros acuáticos en la cuenca del río Coello (Tolima, Colombia). Caldasia 29(1): 177-194.

Asprilla, S., Mosquera, Z. y Rivas, M. 2006. Macroinvertebrados acuáticos como indicadores de calidad ecológica del agua en la parte media del río Cabí (Quibdó, Chocó). Revista de la Asociación Colombiana de Ciencias Biológicas 18: 43-50.

Banco de Occidente. 2009. El Chocó Biogeográfico Colombiano. Libros de la Colección Ecológica. Santafé de Bogotá, Colombia.

Bedoya, G., Cediel, F., Restrepo-Correa, I., Cuartas, C., Montenero, G. y Marín-Cerón, M.I. 2009. Aportes al conocimiento de la evolución geológica de las cuencas Atrato y San Juan dentro del arco Panamá-Chocó. Boletín de Geología 31(2): 61-81.

Bejarano, P.D., Palacio, E.P. y Mosquera, Z. 2006. Evaluación de la calidad del agua por medio de la comunidad de tricópteros durante los períodos de aguas altas y bajas en la quebrada La Francisca, Municipio de Quibdó, Chocó-Colombia. Revista Institucional Universidad Tecnológica del Chocó 25: 59-64. 
Brown, H.P. 1970. Neocylloepus, a new genus from Texas and Central America (Coleoptera: Dryopoidea: Elmidae). The Coleopterists Bulletin 24(1): 1-29.

Brown, H.P. 1981a. Key to the world genera of Larinae (Coleoptera, Dryopoidea, Elmidae), with descriptions of new genera from Hispaniola, Colombia, Australia, and New Guinea. Pan-Pacific Entomologist 57(1): 76-104.

Brown, H.P. 1981b. Huleechius, a new genus of riffle beetles from Mexico and Arizona (Coleoptera, Dryopoidea, Elmidae). Pan-Pacific Entomologist 57(1): 228-244.

Camargo, C. y Rozo, M.P. 2003. Colombian Darien Ephemeroptera. En: Gaino, E., Editor. Research Update on Ephemeroptera and Plecoptera. University of Perugia, Perugia, Italy.

Carter, H.J. y Zeck, A. 1929. A monograph of the Australian Dryopidae. Order Coleoptera. Australian Journal of Zoology 6: 50-72.

Caupaz-Flórez, F., Reinoso, G., Guevara, G. y Villa, F.A. 2006. Diversidad y distribución de la familia Elmidae (Insecta: Coleoptera) en la cuenca del río Prado (Tolima, Colombia). Asociación Colombiana de Limnología Neolimnos 1: 106-116.

Casas-Córdoba, L., Córdoba-Aragón, K.E., Asprilla, S. y Mosquera, Z. 2006. Composición y distribución del Orden Ephemeroptera en los ríos Tutunendo y Catugadó. Quibdó, Chocó-Colombia. Asociación Colombiana de LimnologiaNeolimnos 1: 92-97.

Córdoba, A.K.E., Casas, L., Mosquera, Z. y Asprilla, S. 2007. Composición y variación temporal del orden Ephemeroptera (Insecta) en los ríos Tutunendo y Catugadó. Quibdó (Chocó, Colombia). Revista de la Asociación Colombiana de Ciencias Biológicas 19: 34-41.

Erichson, G.F. 1847. Conspectus Insectorum Coleopterorum, quae in Republica Peruana observata sunt. Archiv für Naturgeschichte 13: 67-201.

González-Córdoba, M. 2016. Elmidae (Insecta: Coleoptera) de Colombia. Tesis de Maestría, Universidad del Valle, Programa de Biología, Cali, Colombia.

González-Córdoba, M., Zúñiga, M. del C. y Manzo, V. 2015a. Riqueza genérica y distribución de Elmidae (Insecta: Coleoptera: Byrrhoidea) en el departamento del Valle del 94 Cauca, Colombia. Biota Colombiana 16(2): 50-74.

González-Córdoba, M., Zúñiga, M. del C., TorresZambrano, N.N. y Manzo, V. 2015b. Primer registro de las especies Neolimnius palpalis Hinton y Pilielmis apama Hinton (Coleoptera: Elmidae: Elminae) para Colombia y la cuenca del río Orinoco. Boletín del Museo de Entomología de la Universidad del Valle 16(1): 27-33.

González-Córdoba, M., Zúñiga, M. del C., Manzo, V., Giraldo, L.P. y Chará, J. 2016. Notelmis Hinton y Onychelmis Hinton (Coleoptera: Elmidae: Elminae) de Colombia: Claves taxonómicas ilustradas. Boletín del Museo de Entomología de la Universidad del Valle 16(2): 1-17.

Grouvelle, A. 1908. Mission de M.F. Geay dans la Guyane (bassin du fleuve Carsevenne). Coléoptères: Helminthidae. Bulletin du Muséum National d'Histoire Naturelle 4: 181-186.

Hinton, H.E. 1934. Miscellaneous studies in the Helminae (Dryopidae, Coleoptera). Revista de Entomología 4(2): 192-201.

Hinton, H.E. 1935. Notes on the Dryopoidea (Coleoptera). Stylop 4(8): 169-179.

Hinton, H.E. 1936. Descriptions of new genera and species of Dryopidae (Coleoptera). Transactions of the Royal Entomological Society of London 85(18): 415-434.

Hinton, H.E. 1940. A monographic revision of the Mexican water beetles of the family Elmidae. Novitates Zoologicae 42(2): 217-396.

Hinton, H.E. 1941a. A synopsis of the American species of Austrolimnius Carter (Col., Elmidae). Entomologist's Monthly Magazine 77: 156-163.

Hinton, H.E. 1941b. New genera and species of Elmidae (Coleoptera). The Transactions of the Royal Entomological Society of London 91(3): 65-104.

Holdridge, L.R. 1996. Ecología basada en zonas de vida. Colección Libros y Materiales Educativos No. 83. Instituto Interamericano de Cooperación para la Agricultura-IICA, San José, Costa Rica.

Instituto de Investigaciones Ambientales del Pacífico-IIAP. 2013. Proyecto Pan estratégico de la macro cuenca del Pacífico Colombiano. Quibdó-Chocó. Informe final.

Jäch, M.A., Kodada, J., Brojer, M., Shepard, W.D. y Čiampor, F. 2016. Coleoptera: Elmidae and Protelmidae. World Catalogue of Insects, (14) Brill, Leiden.

Manzo, V. 2005. Key to the South American genera of Elmidae (Insecta: Coleoptera) with distributional data. Studies on Neotropical Fauna and Environment 40: 201-208.

Manzo, V. 2006. A review of the American species of Xenelmis Hinton (Coleoptera: Elmidae) with a new species from Argentina. Zootaxa 1242: 53-68. 
Manzo, V. 2007. Cinco citas nuevas de Elmidae (Coleoptera) para la Argentina, con la redescripción de Austrolimnius (Telmatelmis) nyctelioides. Revista de la Sociedad Entomológica Argentina 66(1-2): 11-20.

Miranda, G.S., Sampaio, B.H.L. y Passos, M.I.S. 2012. Two new species of Austrolimnius Carter and Zeck (Insecta: Coleoptera: Elmidae) from Southeastern Brazil. Zootaxa 3389: 14-24.

Mittermeier, R.A., Myers, N., Thomsen, J.B., Da Fonseca, G.A.B. y Olivieri, S. 1998. Biodiversity hotspots and major tropical wilderness areas: Approaches to setting conservation priorities. Conservation Biology 12(2): 516-520.

Mosquera, Z., Bejarano, D. y Asprilla, S. 2006. Estudio del Orden Trichoptera (Insecta) en dos ecosistemas lóticos del municipio de Quibdó, Chocó-Colombia. 2006. Asociación Colombiana de Limnología - Neolimnos 1: 85-91.

Mosquera-Murillo, Z. y Córdoba-Aragón, K.E. 2015a. Primer reporte de Miroculis Edmunds (Ephemeroptera: Leotophlebiidae) para el departamento del Chocó, Colombia. Revista de Biodiversidad Neotropical 5(2): 156-158.

Mosquera-Murillo, Z. y Córdoba-Aragón, K.E. 2015b. Caracterización de la entomofauna acuática en cuatro quebradas de la cuenca del río San Juan, Chocó, Colombia. Revista de la Academia Colombiana de Ciencias Exactas, Físicas y Naturales 39(150): 67-76.

Motschulsky, V. 1859. Études Entomologiques. Pt. 8. II. Entomologie spéciale. Insectes des Indes orientales, et de contrées analogues 2: 25-118.

Musgrave, P.N. 1935. Two new Elmidae from Puerto Rico with description of a new genus (Coleoptera). Proceedings of the Entomological Society of Washington 37(2): 32-35.

Myers, N., Mittermeier, R.A., Mittermeier, C., De Fonseca, G.A.B. y Kent, J. 2000. Biodiversity hotspots for conservation priorities. Nature 403: 853-858.

Olson, D.M. y Dinerstein, E. 1998. The global 200: a representation approach to conserving the earth's most biologically valuable ecoregions. Journal of the Society of Conservation Biology 12: 502-515.

Rangel-Ch, J.O. 2004. Colombia diversidad biótica IV: El Chocó biogeográfico-Costa Pacífica. Instituto de Ciencias Naturales, Universidad Nacional de Colombia, Santafé de Bogotá.

Rozo, M.P. y Salinas, L.G. 2016. Inventario del orden Ephemeroptera (Insecta) en la quebrada Coquital, Serranía de Tripogandí, Departamento del Chocó, Colombia. Entomotropica 31(1): 1-13.

Sampaio, B.H.L., Passos, M.I.S. y Ferreira, N. 2015. New species and new records of Xenelmis Hinton (Coleoptera: Elmidae) from Southeastern Brazil. Zootaxa 3936(1): $115-122$.

Sharp, D. 1882. Insecta, Coleoptera. (Haliplidae, Dytiscidae, Gyrinidae, Hydrophilidae, Heteroceridae, Parnidae, Georissidae, Cyathoceridae). En: Godman, F.D. and Salvin, O., Editors. Biologia Centrali-Americana. Taylor y Francis, Londres.

Spangler, P.J. y Santiago, S. 1987. A revision of the Neotropical Aquatic beetle genera Disersus, Pseudodisersus, and Potamophilops (Coleoptera: Elmidae). Smithsonian Contributions to Zoology 446: 1-40.

Spangler, P.J. y Santiago-Fragoso, S. 1992. The aquatic beetle subfamily Larainae (Coleoptera: Elmidae) in Mexico, Central America and the West Indies. Smithsonian Contributions to Zoology 528: 1-74.

Spangler, P.J. y Perkins, P.D. 1989. Revision of the Neotropical aquatic beetle genus Stenhelmoides (Coleoptera: Elmidae). Smithsonian Contributions to Zoology 479: 1-63.

Zúñiga, M. del C. y Cardona, W. 2009. Bioindicadores de calidad de agua y caudal ambiental. En: Cantera-Kintz, J.R., Carvajal-Escobar, Y. y Castro-Heredia, L.M., Editores. Caudal Ambiental: conceptos, experiencias y desafíos. Programa Editorial Universidad del Valle, Colección Libros de Investigación, Santiago de Cali, Colombia.

Fecha de recepción: 10/11/2016

Para citar este artículo: González-Córdoba, M., Zúñiga, M. del C., Mosquera-Murillo, Z. y Sánchez-Vásquez, S. 2016. Riqueza y distribución de Elmidae (Insecta: Coleoptera: Byrrhoidea) en el departamento del Chocó, Colombia. Revista Intropica Vol. 11: 85 - 95 